\title{
Galactic cosmic ray modulation near the heliospheric current sheet
}

Article

Accepted Version

Thomas, S. R., Owens, M. J., Lockwood, M. and Scott, C. J. (2014) Galactic cosmic ray modulation near the heliospheric current sheet. Solar Physics, 289 (7). pp. 2653-2668. ISSN 1573-093X doi: https://doi.org/10.1007/s11207-014-0493-y Available at https://centaur.reading.ac.uk/36500/

It is advisable to refer to the publisher's version if you intend to cite from the work. See Guidance on citing.

Published version at: http://link.springer.com/article/10.1007/s11207-014-0493-y

To link to this article DOI: http://dx.doi.org/10.1007/s11207-014-0493-y

Publisher: Springer

All outputs in CentAUR are protected by Intellectual Property Rights law, including copyright law. Copyright and IPR is retained by the creators or other copyright holders. Terms and conditions for use of this material are defined in the End User Agreement.

\section{www.reading.ac.uk/centaur}

\section{CentAUR}

Central Archive at the University of Reading

Reading's research outputs online 


\title{
Galactic Cosmic Ray Modulation near the Heliospheric Current Sheet
}

\author{
S. R. Thomas ${ }^{1}$ - M. J. Owens ${ }^{1}$. \\ M. Lockwood ${ }^{1}$. C. J. Scott ${ }^{1}$
}

(C) Springer $\bullet \bullet \bullet \bullet$

\begin{abstract}
Galactic cosmic rays (GCRs) are modulated by the heliospheric magnetic field (HMF) both over decadal time scales (due to long-term, global HMF variations), and over time scales of a few hours (associated with solar wind structures such as coronal mass ejections or the heliospheric current sheet, HCS). Due to the close association between the HCS, the streamer belt, and the band of slow solar wind, HCS crossings are often associated with corotating interaction regions where fast solar wind catches up and compresses slow solar wind ahead of it. However, not all HCS crossings are associated with strong compressions. In this study we categorise HCS crossings in two ways: Firstly, using the change in magnetic polarity, as either away-to-toward (AT) or toward-to-away (TA) magnetic field directions relative to the Sun and, secondly, using the strength of the associated solar wind compression, determined from the observed plasma density enhancement. For each category, we use superposed epoch analyses to show differences in both solar wind parameters and GCR flux inferred from neutron monitors. For strong-compression HCS crossings, we observe a peak in neutron counts preceding the HCS crossing, followed by a large drop after the crossing, attributable to the so-called 'snow-plough' effect. For weak-compression HCS crossings, where magnetic field polarity effects are more readily observable, we instead observe that the neutron counts have a tendency to peak in the away magnetic field sector. By splitting the data by the dominant polarity at each solar polar region, we find that the increase in GCR flux prior to the HCS crossing is primarily from strong compressions in cycles with negative north polar fields due to GCR drift effects. Finally, we report on unexpected differences in GCR behaviour between TA weak compressions during opposing polarity cycles.
\end{abstract}

Keywords: Cosmic rays · Heliospheric current sheet $\cdot 22$-year cycle $\cdot$ Energetic particles

\footnotetext{
${ }^{1}$ University of Reading email: s.r.thomas@pgr.reading.ac.uk
} 


\section{Introduction}

In 2009-2010, the heliospheric magnetic field (HMF) intensity reached its lowest value of the space age, which is taken here to be approximately 1965 onwards (Owens et al., 2011; McComas et al., 2012; Lockwood et al., 2012). Simultaneously, near-Earth galactic cosmic ray (GCR) fluxes, inferred from ground-based neutron monitors, peaked at their highest values over the same period (Aslam and Badruddin, 2012; Krymsky et al., 2012), as the HMF modulation effects were weaker (Thomas, Owens, and Lockwood (2013), and references therein). Near-Earth GCR flux can also be inferred from cosmogenic isotopes contained within ice sheets and biomass, allowing the reconstruction of HMF before neutron monitors were in use (e.g. McCracken et al., 2004; Steinhilber, Beer, and Frohlich, 2009; Steinhilber et al., 2010; Usoskin, Bazilevskaya, and Kovaltsov, 2011; Lockwood et al., 2012; Owens, Usoskin, and Lockwood, 2012). On shorter time scales, understanding the heliospheric modulation of GCRs is necessary both to interpret the cosmogenic isotope data and to explain changes seen at Earth, such as those in atmospheric electricity (e.g. Scott et al., 2013), and the effects on modern operational systems such as electronics on satellites and aircraft.

GCR fluxes at Earth are known to be modulated by a variety of different processes within the heliosphere (e.g. McCracken and Ness, 1966). As they travel through the heliosphere they are subject to drift effects, scattering from irregularities, diffusion, and adiabatic deceleration (Parker, 1965). During the 11-year cycle in sunspot number, the Sun's dominant magnetic polarity reverses around the time of solar maximum, which is predicted to have a significant effect on GCR modulation through average particle drift patterns (Jokipii, Levy, and Hubbard, 1977). By convention, the polarity of the solar field $q A$ (where $q$ is the charge on the energetic particle and $A$ is the direction of the solar global field), is taken to be negative when the dominant polar field is inward in the northern hemisphere and outward in the southern, whereas $q A$ is positive if the opposite is true (e.g. Ahluwalia, 1994). Jokipii, Levy, and Hubbard (1977) suggested that particle drifts differ during different $q A$ cycles, with GCR protons reaching Earth from drifting down from the solar poles during $q A>0$ cycles, whereas in $q A<0$ they arrive at Earth down the heliospheric current sheet (HCS). This gives rise to a 22-year cycle in near-Earth GCR flux (Hale and Nicholson, 1925), and has been used to explain successive 'peak' and 'dome' maxima in the neutron count time series.

The HCS separates regions of opposing HMF polarity and lies close to the ecliptic plane around times of solar minimum (Hoeksema, Wilcox, and Scherrer, 1983; Tritakis, 1984), becoming more warped as solar activity increases. The modulation of GCRs by the HCS has been studied in the long term by Paouris et al. (2012) and Mavromichalaki and Paouris (2012). They showed that the long-term variation in GCR modulation can be modelled using a number of solar and heliospheric variables including the tilt angle of the HCS relative to the solar rotation direction, and showed a significant correlation between HCS tilt angle and the GCR modulation parameter during recent solar cycles. 
The HCS passes over Earth a number of times (usually between two and six times) per 27-day rotation (e.g. Smith, 2001). HCS crossings provide an excellent opportunity to sample GCR flux in opposite magnetic polarities at the same stage of the solar cycle and under similar solar wind conditions. However, HCS crossings are often associated with corotating interaction regions (CIRs) (e.g. Tsurutani et al., 1995), due to the HCS's close association with the streamer belt and the band of slow solar wind. These are relevant to the present study as they modulate the GCR flux (for example, Rouillard and Lockwood, 2007). The presence of a CIR in spacecraft measurements is seen as an increase in the solar wind plasma density and magnetic field intensity resulting from the compression of slow solar wind streams by the fast wind behind them. The increased field in this compressed region acts as a barrier to GCR propagation giving enhanced fluxes ahead of it and reduced fluxes behind it, often referred to as the "snow plough effect".

Badruddin, Yadav, and Yadav (1985) separated HCS crossings into awayto-toward (AT) and toward-to- away (TA) magnetic fields, where toward/away sectors are defined as magnetic field lines following a Parker spiral magnetic field directed, towards/away from the Sun, respectively. They considered the period from 1964 to 1976 and split the data into three periods; the solar minimum between cycles 20 and 21, the maximum of cycle 21 , and the minimum between cycles 21 and 22 . For a range of different neutron monitor stations they found that, on average, neutron counts peaked as the HCS crossed Earth and then decreased to a value lower than that before the crossing. Badruddin and Ananth (2003) extended the study period to 1985, essentially including a second solar cycle, and concluded that GCR flux is more strongly affected during $q A>0$ cycles; a finding also noted by El Borie, Duldig, and Humble (1998). They also noted a greater increase in GCRs across AT than TA crossings. Further to this, Richardson, Cane, and Wibberenz (1999) have found that the response of GCRs to modulation by recurrent CIRs is $50 \%$ greater in $q A>0$ than $q A<0$ cycles during two solar minimum periods in the mid-1950s and mid-1990s.

El Borie (2001) compared data from cycles 21 and 22. He first noted differences in apparent propagation characteristics of GCRs between the recovery and declining phases of the solar cycle, including a rigidity dependence of the variation. Furthermore, he notes that GCR flux varies more during toward magnetic field polarity days compared with during away polarity days. However, in each of these investigations, the data available to him only included up to two solar cycles, compared with the four cycles available now. In this study, we aim to add to the two solar cycles used in e.g. El Borie, Duldig, and Humble (1998) and further split the data based upon the strength of the solar wind compression associated with each HCS crossing. The aim is to attempt to separate the 'magnetic barrier' (or 'snow-plough') effect from any effect resulting purely from different magnetic polarities either side of the HCS crossing.

In Section 2 we identify all HCS crossings in the period 1965-2013. This HCS catalogue is used in Section 3 to deduce the average variations in GCR flux over all HCS crossings. 


\section{Identifying Current Sheet Crossings}

In this section, we produce a catalogue of HCS crossings over the period from 1964 to 2012 from the OMNI-2 data set (King and Papitashvili, 2005) of nearEarth solar wind observations. Each crossing is identified by the change in in-ecliptic magnetic field angle, $\phi_{B}$, derived from the Geocentric Solar Ecliptic (GSE) $x$ - and $y$-components from an ideal Parker spiral angle assuming a constant solar wind speed, of approximately $135^{\circ}$ to one of $315^{\circ}$ or vice versa, similar to the method used by El Borie (2001) and Badruddin, Yadav, and Yadav (1985). We do not include HCS crossings in which the magnetic field rotates smoothly or fluctuates between regimes, but rather limit event selection to those that display a sharp transition within a duration of approximately one hour. This reduces the size of the catalogue, but reduces uncertainty in the time of the HCS crossing and means that we are studying quasi-tangential discontinuities (with only a small or zero field threading the structure) rather than rotational discontinuities. The orientation of the HCS crossing (i.e. whether the direction of magnetic field lines change from AT or TA), is deduced from the sign of the magnetic field component, $B_{x}$, in the direction of the Sun from Earth.

A typical HCS crossing is shown in Figure 1. The panels, from top to bottom, show the neutron monitor counts, the in-ecliptic magnetic field angle, the $y$ component of solar wind velocity, the solar wind velocity in the $x$ - direction (this is negative in sign so we take the magnitude to display an increase in speed as being positive), the HMF intensity $|\mathbf{B}|, x$ - component of the HMF and plasma density. Ten days of data centred on 23 December 1999 are shown, with the HCS crossing at time 0 . The neutron monitor data shown were recorded at McMurdo (magnetic latitude of 77.9 South), which has been collecting data since 1964 (e.g. Kruger et al., 2008). McMurdo's location near the south pole is ideal as it provides increased sensitivity to heliospheric modulation effects, due to reduced shielding by the terrestrial magnetic field (Bieber et al., 2004). However, similar results were consistently found at other stations, including northern hemisphere stations such as Thule, Greenland (magnetic latitude of 76.5 North, not shown).

In Figure 1 we show the variation of each parameter in hourly values for five days each side of the crossing. In the top panel we see a steady increase in the neutron monitor counts, until approximately the time of the crossing, where it decreases slightly before levelling off. By comparing the in-ecliptic magnetic field angle to the ideal Parker spiral angles (computed assuming a steady solar wind speed of $400 \mathrm{~km} \mathrm{~s}^{-1}$ and shown in orange, this angle does not change much for typical solar wind speeds), the second panel from the top shows the HCS crossing as a rapid change from $135^{\circ}$ to $315^{\circ}$. The bottom panel shows that $B_{x}$ changes from negative to positive and so this is an AT crossing. The $y$ - component of the solar wind velocity is given in the third panel and shows a reversal from negative velocity to positive across the HCS, consistent with the flow deflection at a stream interface (Borovsky and Denton, 2010). The magnitude of the radial solar wind velocity, $v_{x}$, increases over the crossing, as does $B_{x}$ in agreement with the spiral angle increase. We see large peaks in the HMF intensity and the plasma density at the HCS crossing, associated with the compression region.

We searched for events with a similar reversal in spiral angle, throughout the whole one hour resolution OMNI-2 dataset and found a total of 1950 events. 


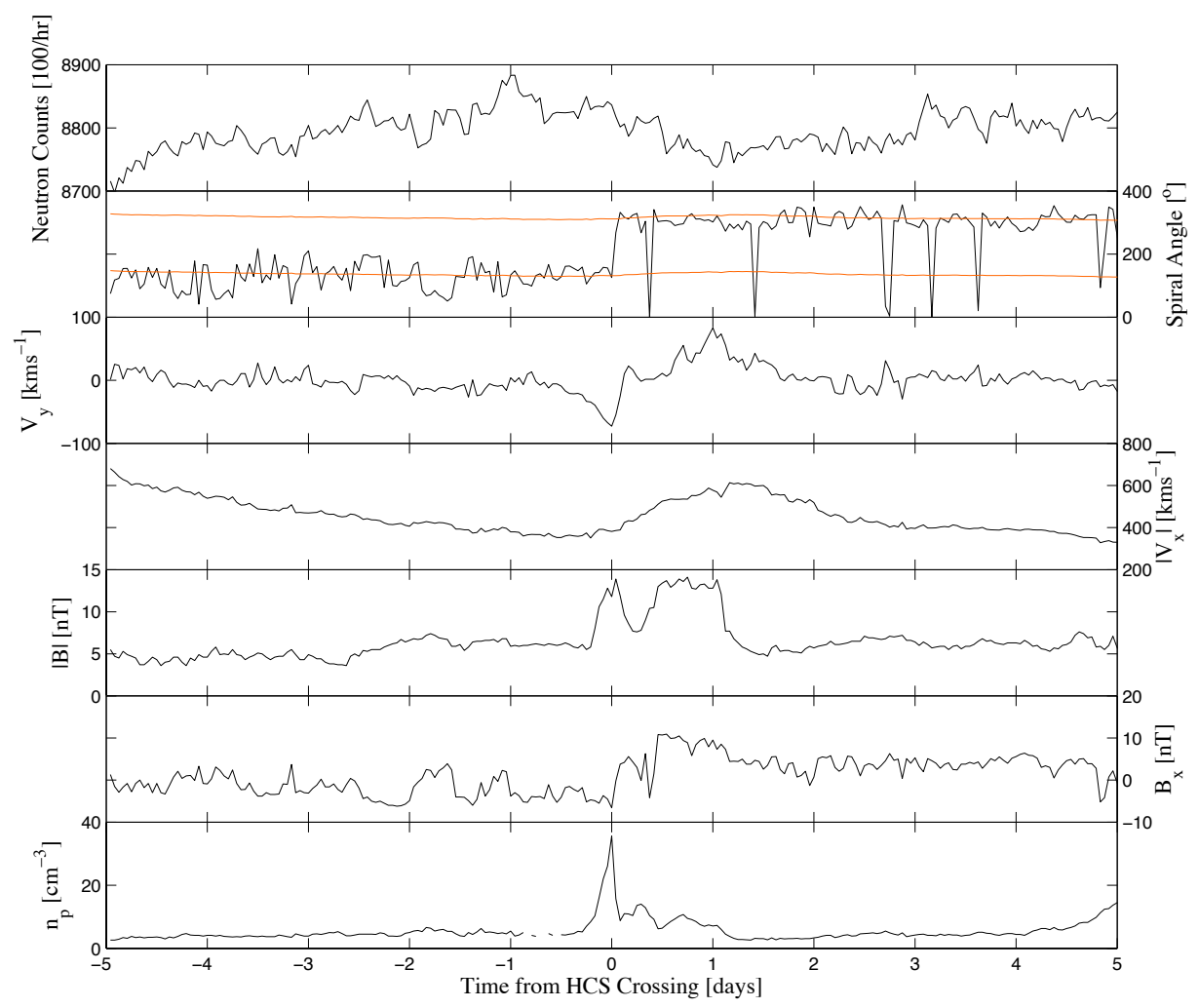

Figure 1. A typical HCS crossing centred on 23 December 1999. From top; neutron counts, in-ecliptic magnetic angle of magnetic field, solar wind velocity component in $y$ - direction, solar wind speed in $x$-direction, heliospheric magnetic field magnitude, HMF in $x$ - direction, and plasma density. The vertical dashed line indicates the HCS crossing defined by the change in magnetic angle. The horizontal orange lines display the ideal in-ecliptic magnetic field angles.

Including data gaps, this equates to an equivalent of one HCS crossing every nine days. However, removing data gaps and unclear HCS crossings due to extended rotations in the in-ecliptic magnetic field direction (perhaps owing to the presence of coronal mass ejections at the HCS; e.g. Crooker et al., 1998) reduced the event list to 402 HCS crossings, approximately one event per 45 days. Thus, the more conservative criterion for event identification we have adopted means that the rate of events studied is much lower than that used in El Borie (2001) who compiled 71 events in a three-year period and 108 in four years during a later period for their study, and also than Badruddin, Yadav, and Yadav (1985) and Badruddin and Ananth (2003) who restricted their catalogue to those where the polarity did not change for at least five days before and after the HCS crossings. 


\section{Cosmic Ray Variations Associated with Current Sheet Crossings}

We now look at solar wind and GCR variations across the HCS statistically. Figure 2 shows a superposed epoch study (also called a "Chree analysis" or a "composite"), using the HCS crossing as the zero epoch time, denoted $t_{0}$, and showing the percentage change in neutron counts (discussed further below), the solar wind speed, plasma density and HMF field strength as a function of epoch time, $t_{\mathrm{e}}\left(=t-t_{0}\right)$, between five days before and five days after the HCS crossing. The mean variation is shown as the blue lines in Figure 2.

The significance of any variations in the means are tested by a Monte-Carlo approach, in which we repeat the exact same analysis, but for 402 randomly selected zero epoch times, rather than 402 HCS crossing times. This process is repeated 1000 times to generate 1000 random means at every epoch time. The resulting Monte-Carlo mean is shown by the black line in Figure 2, and the bounds between which $90 \%$ of the random epoch time means are contained are displayed as the shaded region (i.e., the upper and lower bands are the $5 \%$ and $95 \%$ confidence intervals). Thus HCS crossings generate variations in the observed properties which are significant at every epoch time (at the $95 \%$ confidence level) above random fluctuations at times when the observed mean lies outside of the shaded band. This test has been applied on all further figures in the article.

We first concentrate on the heliospheric parameters shown in Figure 2. The significant peaks in the plasma density (top right) and HMF intensity (bottom left) herald the presence of the compression regions expected by the association of the HCS with CIRs. Here, the typical peak in density is approximately 14 $\mathrm{cm}^{-3}$ compared with the average background density of $6 \mathrm{~cm}^{-3}$ and the HMF intensity increases from approximately $5.2 \mathrm{nT}$ to $8.2 \mathrm{nT}$. We note the large reduction in radial solar wind speed, to an average of $360 \mathrm{kms}^{-1}$ before a steep rise to $460 \mathrm{kms}^{-1}$. This clearly shows the presense of a transition from slow solar wind to fast wind as the HCS passes the spacecraft. Similar patterns at stream interfaces, where slow proceeds fast wind, were shown in these variables by Crooker and McPherron (2012).

Neutron monitor counts show solar cycle variations much larger than typical variations across the HCS. Therefore, in order to compose a superposed epoch analysis of GCR flux variations associated with HCS crossings, it is necessary to normalise the neutron counts. We take a background value of each parameter defined as the mean of hourly values from five days before to five days after the HCS crossing time, but excluding twelve hours each side of the crossing itself. From this, we compute the percentage change in neutron monitor counts relative to the background. Any changes above $20 \%$ are attributed to large solar energetic particle (SEP) events and removed from the data set (Barnard and Lockwood, 2011 ). However, this only reduces the data size by approximately $0.5 \%$. Figure 2 (top left) shows a superposed epoch analysis of percentage change in neutron counts relative to the background level for all HCS crossings in the four solar cycles considered in the study. On average we see a peak in neutron counts of $0.35 \%$ over the background just before the HCS crosses the spacecraft, which is considerably greater than the $95 \%$ confidence level. Following the HCS crossing 

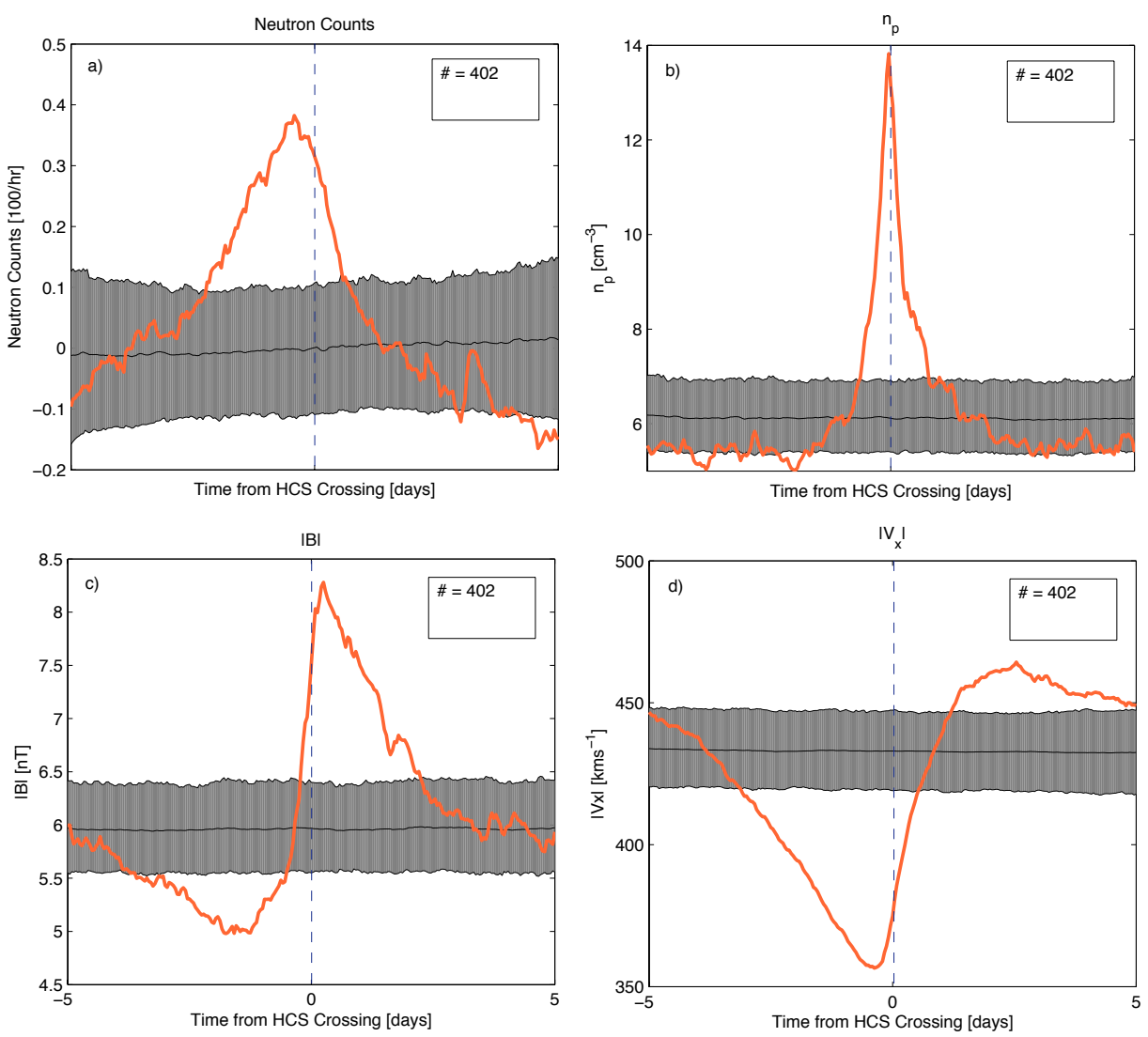

Figure 2. Superposed epoch analysis for all HCS crossings within the four solar cycles (orange lines) showing (a) percentage change in neutron counts, (b) plasma density, (c) magnitude of the heliospheric magnetic field, and (d) radial solar wind velocity. The black lines are the means of the Monte-Carlo analysis using random event times and the shaded regions are the $95 \%$ and $5 \%$ confidence bands. The vertical dashed lines show the zero epoch time of the HCS crossing $\left[t_{0}\right]$. The number of events are given in the boxes in the top right of each panel.

the neutron counts are depressed but this only exceeds the $95 \%$ significance level after about four days following the crossing.

In general, on long time-scales, neutron counts are known to be modulated by the HMF. Therefore, if this applied on all time and spatial scales, the profile of the neutron counts would appear as the inverse of the magnitude of the HMF. However, the peak and trough of the neutron counts are not located at the same time as the trough and peak of the HMF strength, respectively. This behaviour is due to the snow-plough effect where, as a region of compressed magnetic field propagates out through the heliosphere, it pushes a region of enhanced energetic particle flux in front of it, with a region of depleted GCR flux immediately behind it (e.g. Richardson, 2004).

Figure 3 shows the corresponding results for the same dataset split into AT and TA events. Here Figure 3a shows the AT HCS crossings and Figure 3b 


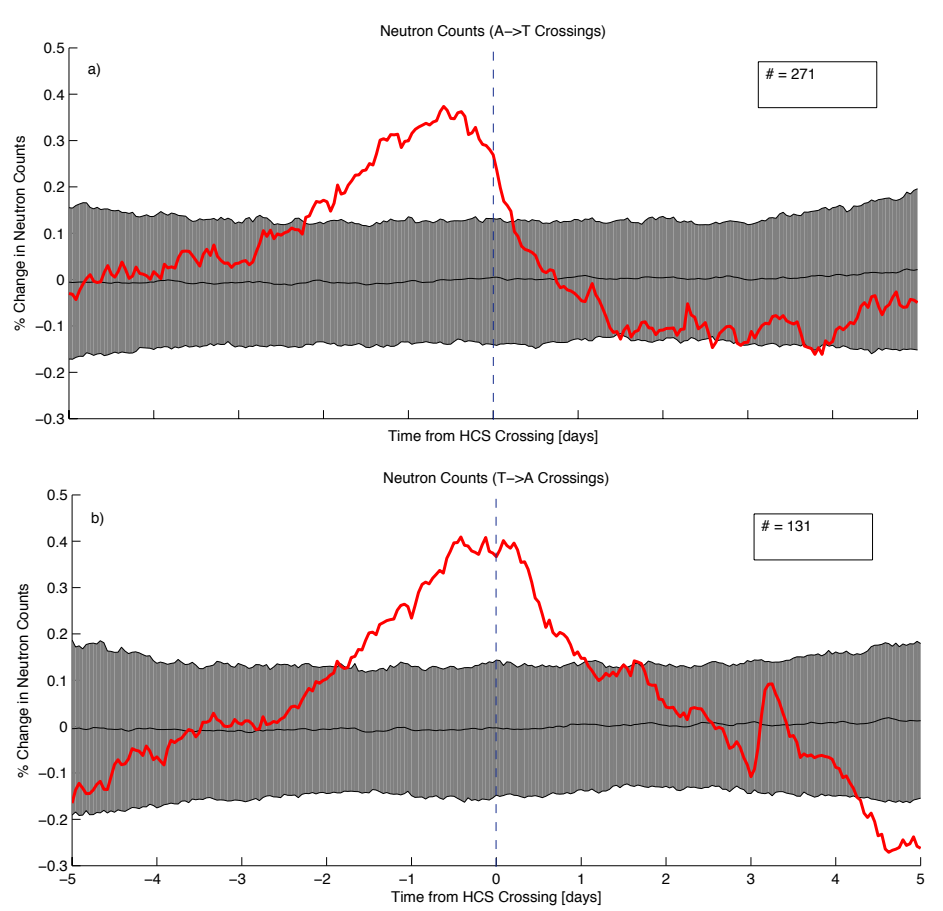

Figure 3. Superposed epoch analyses of the percentage change in neutron counts from the background (red lines): (a) means of away to toward HCS crossings and (b) toward to away HCS crossings. The black lines again are the means of the Monte Carlo analysis using random event times and the shaded region is the $95 \%$ confidence band, and the vertical line is the zero epoch time.

shows TA crossings. Again, a Monte-Carlo analysis is applied and the 95\% confidence interval shown by the shaded region. The heliospheric parameters are not included here but show the same patterns as in Figure 2 (i.e. there are no systematic differences in the solar wind compression characteristics for TA and AT events).

Comparing Figures $3 \mathrm{a}$ and $3 \mathrm{~b}$ we note differing behaviour in GCR flux between the AT and TA cases. The GCR flux in the AT case shows a build up in GCR flux peaking approximately a day before $t_{0}$, whereas the GCR flux peaks almost symmetrically over $t_{0}$ in the TA case. There are also notable differences in the days following the HCS crossing. The GCR flux after an AT HCS crossing falls off steeply to an approximately $95 \%$ significant depletion of GCRs from one to four days after the crossing. However, after the TA HCS crossings, the GCR flux decreases more gradually and does not reach a $95 \%$ significance level depletion until over four days after the crossing. 


\section{Effect of Solar Wind Compression on Neutron Counts}

As discussed above, HCS crossings are often associated with a transition between slow and fast solar wind (e.g. Thomas and Smith, 1981). The resulting compression, which often forms a CIR, can frequently result in increased plasma density and HMF intensity. Thus, the local strength of the compression front in the heliosphere can be estimated on the basis of the plasma density and magnetic field intensity enhancement observed in near-Earth space. We therefore here divide the HCS into "strong" (SCC) and "weak" (WCC) compression crossings on this basis.

For each HCS crossing, the magnitude of the maximum in plasma density within 2.5 days of the HCS crossing is compared with the background value, defined in the same manner as the neutron counts background, above. A plasma density greater than three times the background value is taken to constitute a strong compression, whereas a plasma density less than three times the background is here classed as a weak compression. Splitting the data in this way results in 271 strong compressions and 131 weak compressions.

Superposed epoch analyses of the plasma density and magnetic field intensity for SCCs (top panels) and WCCs (bottom panels), are displayed in Figure 4. The coloured lines here show the mean value of each epoch and the shaded region and black lines are again the results of a Monte-Carlo analysis with $95 \%$ of 1000 randomly selected events within the shaded region.

Events that we define as SSCs here (Figures 4a and 4b) have large, sharp peaks in plasma density, $n_{\mathrm{p}}$, and a signficant increases in HMF intensity, $|\mathbf{B}|$, well outside of the $95 \%$ significance level. However, the events in Figure 4c found to have low plasma density enhancements, also have low magnetic field intensity enhancements (shown in Figure 4d). Here, a weak depression before the HCS crossing is seen to evolve slowly to a weak enhancement after it. The depression and enhancement in the HMF both still exceed the $95 \%$ confidence level but they crucially have a much smaller magnetic field enhancement at around the zero epoch time. In other words, the WCCs are, unlike the SCCs, not associated with a strong magnetic barrier. Reducing the threshold for weak events any further would mean that there is not a large enough sample, but note that the theshold used means a magnetic barrier is present in WCC cases, on average, albeit a much weaker one.

Figure 5 repeats the analysis of Figure 3, but subdivides the dataset into strong and weak compression HCS crossings. The same format is applied as from previous figures. Figures 5a and 5b show AT and TA SCCs whereas Figures 5c and $5 \mathrm{~b}$ show AT and TA WCCs, respectively. Note that compared with Figure 3, the width of the $95 \%$ confidence bands has increased, due to the reduced sample size from the subdivision of the dataset.

There are a number of points of note. Firstly, Figure 3a shows that in the AT case, a peak in neutron counts occurs approximately a day before the HCS crossing. The AT HCS crossings in Figures 5a (strong compressions) and 5c (weak compressions) both show the same characteristics, where the peak in neutron counts occurs before the HCS crossing (i.e. when $t<t_{\mathrm{e}}$ ). However, Figure $3 \mathrm{~b}$ showed a peak in neutron counts for the TA case which is approximately 

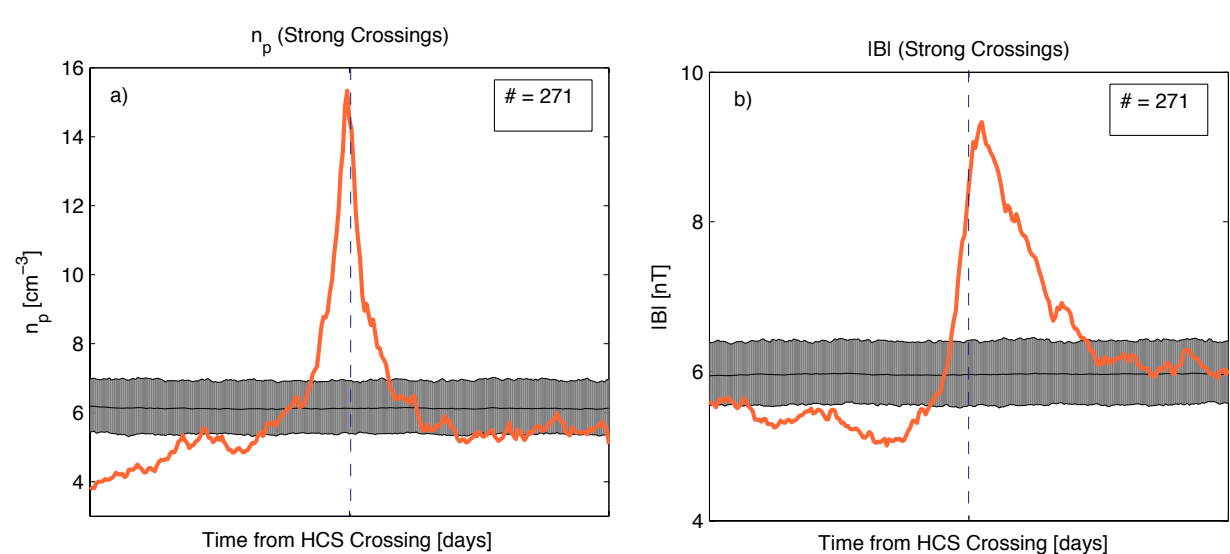

$n_{p}$ (Weak Crossings)
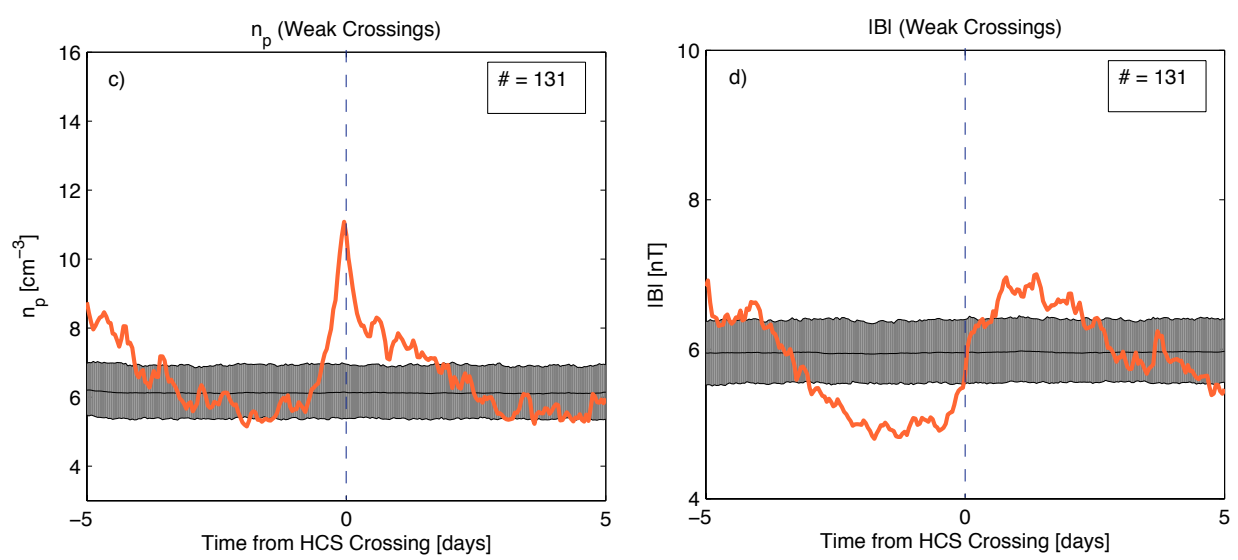

Figure 4. Left: Means of solar wind plasma density (orange lines), $n_{\mathrm{p}}$, during: (a) strong compression HCS crossings and (c) weak compression HCS crossings. HMF intensity during: (b) strong compression HCS crossings and (d) weak compression HCS crossings. The Monte-Carlo analyses are again shown. The number of events for each row is given in the top-right of each plot and the vertical lines are the zero epoch times.

symmetrical across $t_{0}$. Figures $5 \mathrm{~b}$ and $5 \mathrm{~d}$ do not peak at the same $t_{\mathrm{e}}$. Therefore, it is clear that Figure $3 \mathrm{~b}$ is made from a combination of an increase before the HCS crossings in the strong compression case (Figure 5b) and a increase in neutron counts after the crossing in the weak compression case (Figure $5 \mathrm{~d}$ ), as these curves are approximately a mirror image of each other across the HCS crossing.

Secondly, the SCCs (Figures 5a and 5b) both have similar characteristics. The neutron counts are seen to increase to a maximum before the HCS crossing and then to decrease across it to a minimum later in the time period. Again, the neutron counts are significantly depleted between one and four days after the HCS crossing in the AT case (Figure 5a) but are seen to drop out of the $95 \%$ significance after approximately three days in the TA case (Figure 5b).

The WCCs show a different pattern, however. By largely removing the strong magnetic barrier associated with strong compressions, we can better observe 

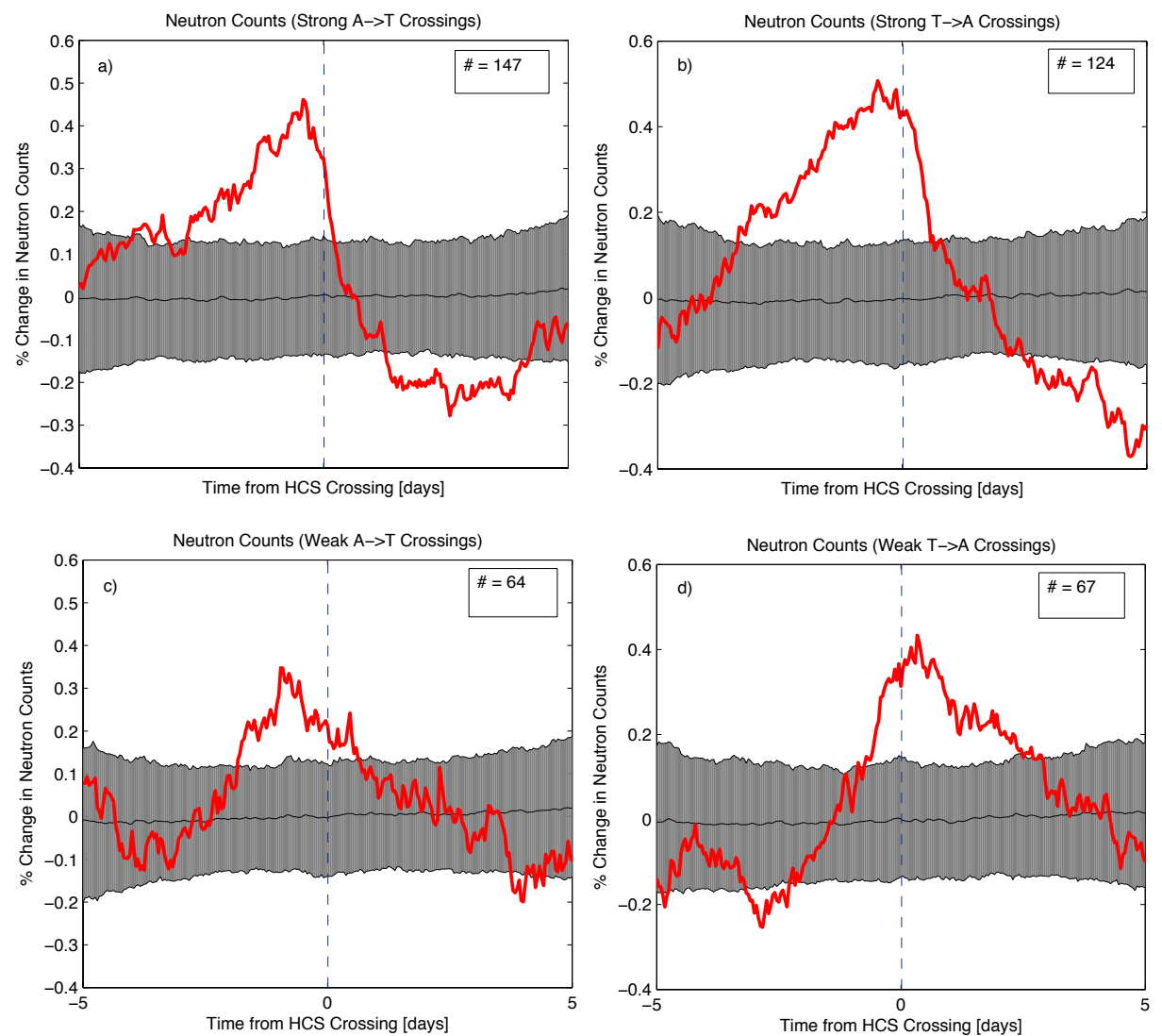

Figure 5. Red lines display the percentage change in neutron counts across HCS crossings. Strong compressions are shown in the top panels ( $a$ and b) and weak compressions at the bottom (c and d). Again the black line and shaded regions are the mean of the Monte-Carlo runs. The number of events in each epoch is given in the top right of each panel and the vertical line is the zero epoch time.

any effect of the change in magnetic polarity. Figure 5c shows a significant enhancement in GCR flux prior to the AT HCS crossing, which gradually reduces throughout the rest of the time period. Figure 5d, however, shows a large peak in neutron counts after the TA HCS crossing. This peak is approximately a day later than for the AT case, although the increase is greater in the TA case from a significant depletion at three days before the HCS crossing. A result of this is that there is, in general, an increase in GCR flux in the away magnetic field sector, within the vicinity of the HCS.

Heliospheric parameters such as the HMF intensity were also split into AT and TA HCS crossings and it was found that there are no difference in the timing of the peak in HMF intensity between these cases (not shown). Therefore, any difference between AT and TA HCS crossings cannot be directly attributed to any difference in the HMF or plasma density and so is associated with the field polarities. 


\section{Effect of Solar Polarity Reversals}

In this section, we investigate GCR variations across the HCS in different solar polarity cycles. The difference in direction of the propagation of GCRs through the heliosphere, as suggested by Jokipii, Levy, and Hubbard (1977), would be expected to cause a difference in their behaviour across the HCS. During $q A<$ 0 cycles, the northern polar magnetic field is toward the Sun and GCRs are predominantly reaching Earth along the HCS from the outer heliosphere. In this case, a strong compression region at the HCS would be expected to shield Earth from the incoming GCRs after the compression region has passed over the Earth, because then the magnetic barrier is between the Earth and the GCR source. However, during $q A>0$ cycles, the northern polar field is away from the Sun and GCRs predominantly arrive at Earth from over the solar magnetic poles. In this case, the shielding may be earlier as the barrier approaches Earth and would not be a large factor after is has passed the Earth.

To define the polarity, $A$, we use the observed solar polar reversal times as defined in Thomas, Owens, and Lockwood (2013). Figures 6 and 7 show all of the events from Figure 5, but split into the $q A<0$ and $q A>0$ polarity cycles, respectively, with each of the plots including two polarity cycles worth of data.

We first note that for all SCCs during $q A<0$ and $q A>0$ polarity cycles (Figures 6a, 6b, 7a, and 7b), that we see the snow-plough effect. However, we do note some differences between different magnetic polarity SCCs. Note that here we only discuss features that exceed the $95 \%$ confidence level at some point in their evolution.

- For $q A<0$ polarity cycles (Figures $6 \mathrm{a}$ and $\mathrm{b}$ ), the sense of the HCS crossing (i.e. whether it was AT or TA crossing), does not affect GCR variations. However, for $q A>0$ cycles (Figures 7a and b), TA and AT crossings are substantially different in behaviour, as TA crossings appear to be a much greater barrier to GCRs than we observes at AT crossings.

- For AT events, there is a polarity cycle effect, where the "snow-plough effect" is much stronger during $q A<0$ polarity cycles (Figure 6a) than during $q A>0$ cycles (Figure $7 \mathrm{a}$ ).

- For TA events, we also see a polarity cycle effect, although this is different in behaviour to the effect seen between AT events. $q A<0$ polarity cycles show a build up similar than for the AT case (Figure 6b). However, during $q A>0$ polarity cycles we see a significant enhancement in GCR flux prior to the HCS and lasting from 3.5 days before the HCS crossing to a day afterwards (Figure $7 \mathrm{~b}$ ).

For WCCs, in general, the difference in solar polarity and the sense of the HCS crossings all seem to affect GCR variations. We shall now discuss some key features of the WCCs (Figures 6c, 6d, 7c, and 7d).

- AT HCS crossings are an exception to this rule (Figures $6 \mathrm{c}$ and $7 \mathrm{c}$ ), in that the solar polarity does not have an obvious effect. In both cases there is a GCR variation in agreement with a weak snow-plough effect due to the associated weak magnetic field enhancement. 

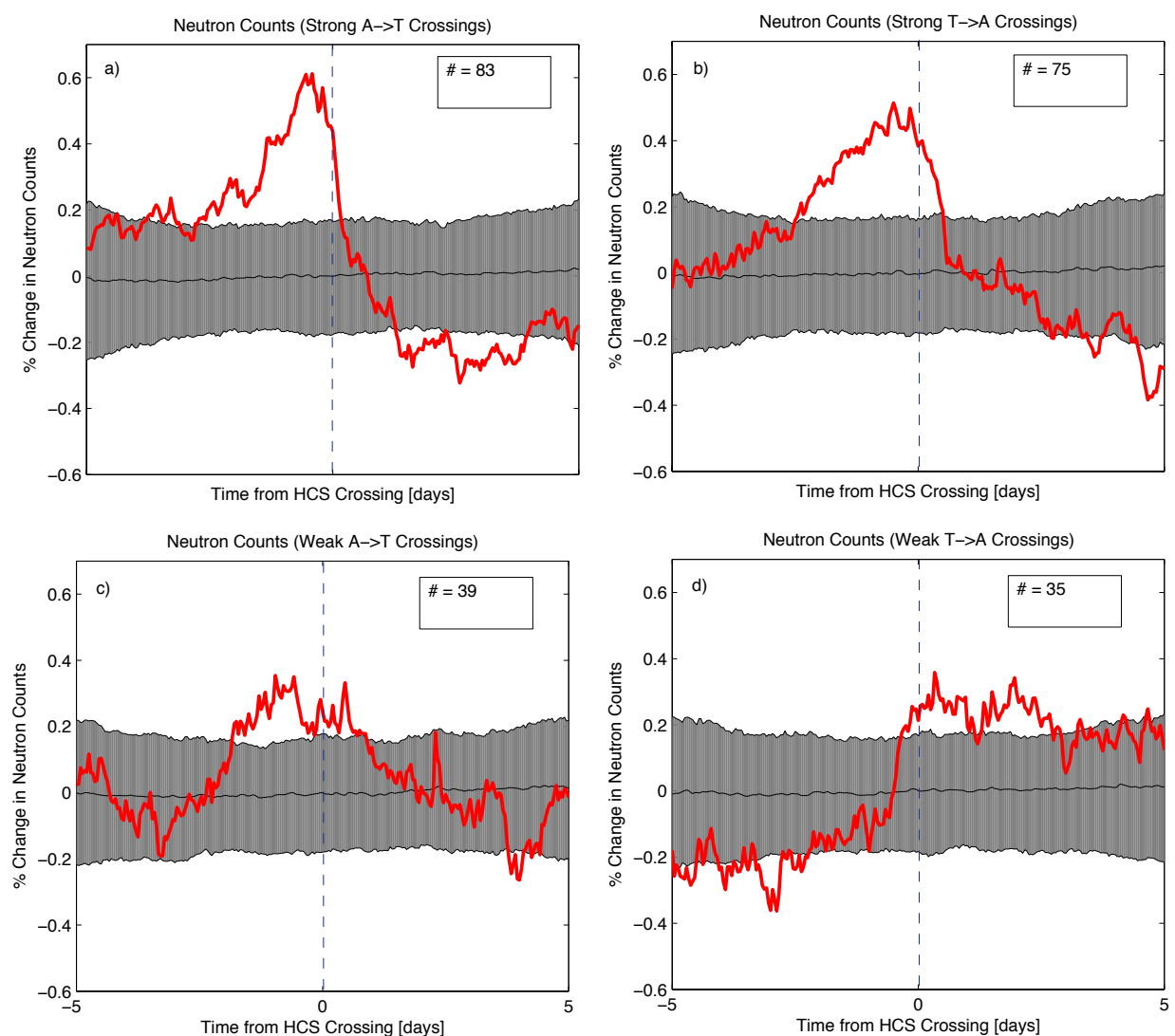

Figure 6. A superposed epoch analysis of HCS crossings during $q A<0$ polarity cycles. The format and events used are both the same as used for previous figures.

- However, for TA crossings during $q A>0$ polarity cycles (Figure $7 \mathrm{~d}$ ), there is a strong enhancement in GCR flux, which is roughly symmetrical about the HCS crossings. This enhancement is roughly in agreement with the enhancement in Figure 7b, but does not begin so early with respect to the HCS.

- For TA crossings during $q A<0$ polarity cycles (Figure $6 \mathrm{~d}$ ), there is a very different variation in the behaviour of GCRs. Here there is a significant depletion to the $95 \%$ level in GCRs from five to three days before the HCS crossing, which increases in almost a step-change just prior to the crossing to a significant enhancement in GCR flux from $t_{0}$ to three days after the crossing.

- Finally, we note that $q A<0$ cycles do show a tendency for greater GCR flux in away sectors. However, this difference is much less pronounced than in $q A>0$ cycles, where GCR flux enhancement is almost symmetric across the HCS crossing. 

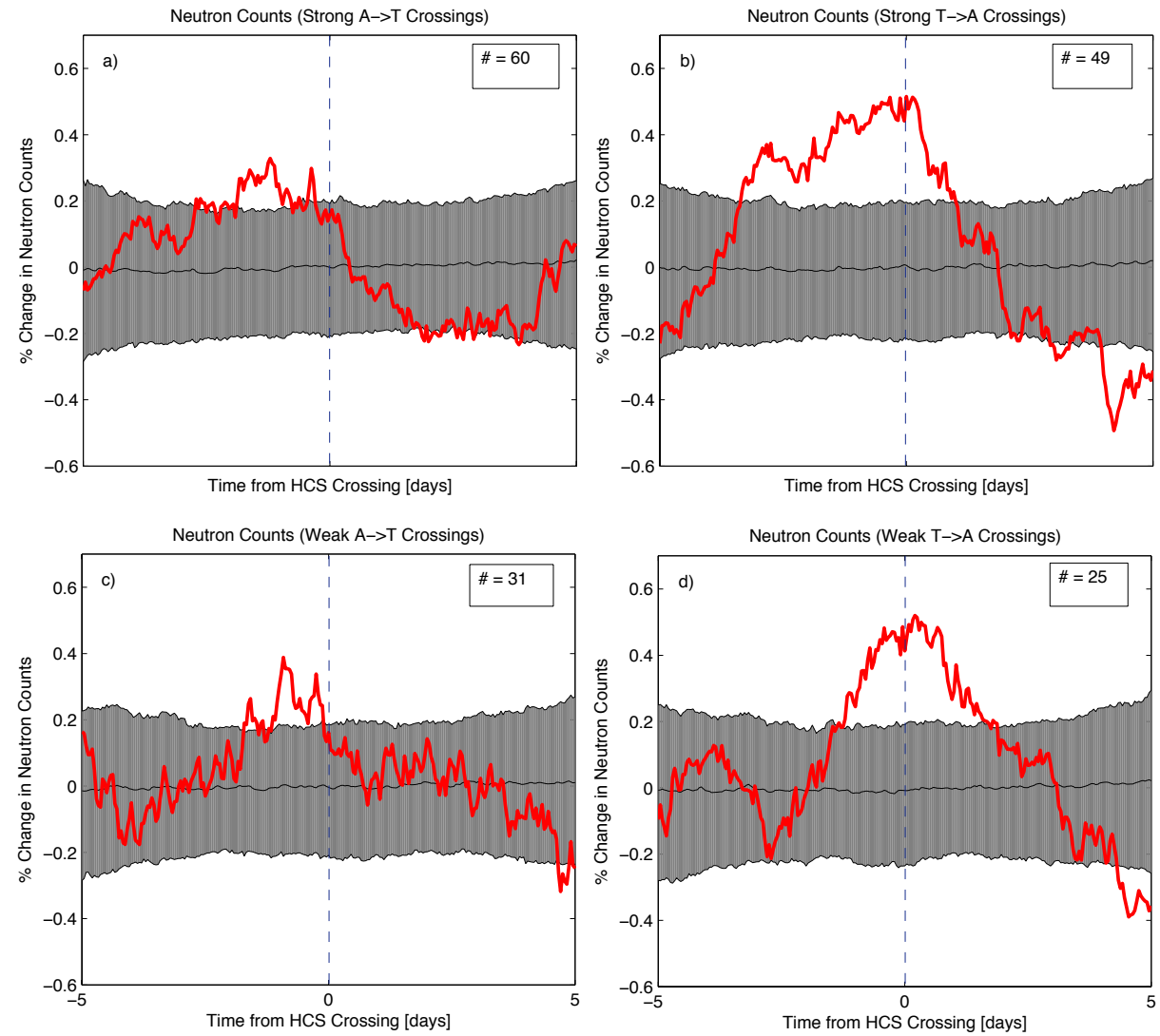

Figure 7. A superposed epoch analysis of HCS crossings during $q A>0$ polarity cycles. Format is again the same as previous figures.

\section{Discussion and Conclusions}

To analyse the behaviour of GCRs across heliospheric current sheet (HCS) crossings we have collected 402 clear instances where the HCS has crossed Earth. We have used superposed epoch analyses to look at small but systematic trends that may otherwise be swamped by event-to-event variability and noise when considering a single case study. Approximately half of the identified HCS crossings are away to toward (AT) with the other half being toward to away (TA) magnetic field directions. We have also divided these events into "strong" and "weak" compression HCS crossings. Splitting the data in this way allows us to separate the effects of large compression regions, which act as a barrier to GCR propagation, and changing magnetic polarity from AT or TA. We shall now summarise our key findings and discuss their implications.

- When splitting the data into AT and TA HCS crossings, we find that the GCR flux at AT HCS crossings peaks approximately a day before the HCS 
crossings for AT crossings but is centred over the HCS for TA crossings. There is no variation in the timing of the peak in the intensity of the heliospheric magnetic field or plasma density between AT and TA HCS crossings to account for this difference.

- Strong compression HCS crossings (SCCs) always display the "snow-plough effect", independent of HCS crossing being AT or TA, and in general show a greater variation than weak compression crossings (WCCs). This effect is associated with CIRs, where GCR flux is know to peak shortly before the HCS crossing, followed by a large depletion in GCRs after the barrier has passed through owing to the scattering off inhomogeneities within the CIR as it moves out through the heliosphere (Richardson, 2004). These general results are consistent with previous findings (e.g. Badruddin, Yadav, and Yadav, 1985; El Borie, Duldig, and Humble, 1998; Richardson, 2004).

- To reduce the dominant effect of the barrier in SCCs, WCCs need to be considered when observing the differing behaviour of GCR flux between AT and TA HCS crossings. SCCs show similar behaviour independent of the sense of the HCS crossing, but for WCCs, AT and TA crossings are not the same. The peak in GCR flux occurs after HCS crossings in the TA case but is seen before the HCS in the AT case. We propose that this different between toward and away sectors is due to the ease in which the GCRs can access magnetic field lines in each polarity. GCR drift effects as described by Jokipii, Levy, and Hubbard (1977), however, do not appear to be the direct cause, as there are more GCRs within the away sector independent of polar polarity.

- When splitting the data further into polarity cycles, it is seen that all SCCs show the "snow-plough effect" to some degree. For AT events, we see a much greater variation in GCR flux across the HCS during $q A<0$ polarity cycles than for those during $q A>0$ cycles. This is in agreement with drift effects as described by Jokipii, Levy, and Hubbard (1977). During $q A<0$, GCRs drift to Earth from the outer heliosphere down the HCS. As the HCS approaches, GCR flux is likely to increase due to scatter from the approaching magnetic field enhancement. However, as GCRs drift from over the solar poles to Earth during $q A>0$ cycles, then this effect is unlikely to be as strong.

- For TA events, we also find a polarity cycle difference, but this is different to that seen for AT events. One would expect a larger "snow-plough effect" from HCS crossings during $q A<0$ than $q A>0$ polarity cycles from drift effect, but instead we see a large and long-lasting enhancement from 3.5 days to the time of the crossing during $q A>0$ polarities. Although drift effects appear not to be the cause, the reason for this enhancement is not clear.

- For AT WCCs in both solar magnetic polarities, we see evidence of a weak "snow-plough effect" due to the weak but significant increase in the heliospheric magnetic field intensity.

- TA WCCs show a very different variation in GCR flux depending on solar polarity. During $q A>0$ polarity cycles, these show an almost symmetrical, large peak across the HCS. The overall pattern is not similar to the AT 
case but is compared to the SCCs case, but without the early increase in GCR flux. On the other hand, during $q A<0$ polarity cycles, we observe a strong step increase in GCR flux from before the HCS to after it, with more GCRs present within the away-from-Sun magnetic field lines. The causes of these behaviours is not clear, although it is worth noting that there are only 35 events in these superposed epoch analyses and so sample sizes have decreased but the division of the data.

- Although we agree with key conclusions of previous studies (e.g. El Borie, Duldig, and Humble, 1998; Richardson, Cane, and Wibberenz, 1999), we find a number of notable differences. For example, El Borie, Duldig, and Humble, 1998 found a larger percentage increase across the HCS crossing than we report, with the peak in GCR flux occurring approximately a day later for AT than TA HCS crossings, where in fact we note the opposite behaviour. Our results also differ from those of Badruddin and Ananth (2003) and El Borie, Duldig, and Humble (1998) as we do not see evidence of a greater degree of GCR modulation during $q A>0$ cycles than during $q A<0$ cycles. Furthermore, we note that for TA WCCs, GCR flux is considerably greater in the away sector during $q A<0$ cycles but there is little difference during $q A>0$ cycles. These differences may arise as we have been very conservative when selecting HCS crossings and consequently have selected fewer HCS crossing events per year. However this has been comprensated for, in terms of numbers of events, because we have considered a longer period including four polarity cycles, compared to their two or three available cycles at the time.

Acknowledgements We are grateful to the Space Physics Data Facility (SPDF) of NASA's Goddard Space Flight Centre for combining the data into the OMNI 2 data set which was obtained via the GSFC/SPDF OMNIWeb interface at http://omniweb.gsfc.nasa.gov. We also thank the Bartol Research Institute of the University of Delaware for the neutron monitor data from McMurdo, which is supported by NSF grant ATM-0527878. The work of SRT is supported by a studentship from the UKs Natural Environment Research Council (NERC).

\section{References}

Ahluwalia, H.S.: 1994, Cosmic ray transverse gradient for a Hale Cycle. J. Geophys. Res. 99, $23515-23521$.

Aslam, O.P.M., Badruddin: 2012, Solar modulation of cosmic rays during the declining and minimum phases of Solar Cycle 23: Comparison with past three solar cycles. Solar Phys. 279, $269-288$.

Badruddin, Ananth, A.G.: 2003, Variation of cosmic ray intensity with angular distance from Earth to the current sheet. 28th Int. Cosmic Ray Conf., 3909-3912.

Badruddin, Yadav, R.S., Yadav, N.R.: 1985, Intensity variation of cosmic rays near the heliospheric current sheet. Planet Space Sci. 2, 191-201.

Barnard, L., Lockwood, M.: 2011, A survey of gradual solar energetic particle events. J. Geophys. Res. 116, A05103.

Bieber, J.W., Clem, J.M., Duldig, M.L., Evenson, P.A., Humble, J.E., Pyle, R.: 2004, Latitudinal survey observations of neutron monitor multiplicity. J. Geophys. Res. 109, A12106.

Borovsky, J.E., Denton, M.H.: 2010, Solar wind turbulence and shear: A superposed-epoch analysis of corotating interaction regions at 1AU. J. Geophys. Res. 115, A12228.

Crooker, N.U., McPherron, R.L.: 2012, Coincidence of composition and speed boundaries of the slow solar wind. J. Geophys. Res. 117. doi:A09104. 
Crooker, N.U., McAllister, A.H., Fitzenreiter, R.J., Linker, J.A., Larson, D.E., Lepping, R.P., Szabo, A., Steinberg, J.T., J., L.A., Mikic, Z., Lin, R.P.: 1998, Sector boundary transformation by an open magnetic cloud. J. Geophys. Res. 103, 26859-26868.

El Borie, M.A.: 2001, Cosmic ray intensities near the heliospheric current sheet throughout three solar activity cycles. J. Phys. G. 27, 773-785.

El Borie, M.A., Duldig, M.L., Humble, J.E.: 1998, Galactic cosmic ray modulation and the passage of the heliospheric current sheet at Earth. Planet. Space Sci. 46, 439-448.

Hale, G.E., Nicholson, S.B.: 1925, The law of Sun-spot polarity. Astrophys. J. 62, 270-300.

Hoeksema, J.T., Wilcox, J.M., Scherrer, P.H.: 1983, The structure of the heliospheric current sheet - 1978-1982. J. Geophys. Res. 88, 9910-9918.

Jokipii, J.R., Levy, E.H., Hubbard, W.B.: 1977, Effects of particle drift on cosmic ray transport. I. General properties, application to solar modulation. Astrophys. J. 213, 861-868.

King, J.H., Papitashvili, N.E.: 2005, Solar wind spatial scales in and comparisons of hourly wind and ACE plasma and magnetic field data. J. Geophys. Res. 110, A02104.

Kruger, H., Moraal, H., Bieber, J.W., Clem, J.M., Evenson, P.A., Pyle, K.R., Duldig, M.L., Humble, J.E.: 2008, A calibration neutron monitor: energy response and instrumental tempaerature sensitivity. J. Geophys. Res. 113, A08101.

Krymsky, G.F., Krivoshapkin, P.A., Gerasimova, S.K., Gololobov, P.Y., Grogor'ev, V.G., Starodubtsev, S.A.: 2012, Heliospheric modulation of cosmic rays in solar cycles 19-23. Astronomy Lett. 9, 609-612.

Lockwood, M., Owens, M.J., Barnard, L., Davis, C.J., Thomas, S.R.: 2012, What is the Sun up to? Astron. Geophys. 53, 3.9-3.15

Mavromichalaki, H., Paouris, E.: 2012, Long term cosmic ray variability and the CME-index. Adv. Astron, 607172

McComas, D.J., Dayeh, M.A., Allegrini, F., Bzowski, M., de Majistre, R., Fujiki, K., Funsten, H.O., Fuselier, S.A., Gruntman, M., Janzen, P.H., Kubiak, M.A., Kucharek, H., Livadiotis, G., Moebius, E., Reisenfeld, D.B., Reno, M., Schwadron, N.A., Sokol, J.M., Tokumaru, M.: 2012, The first three years of IBEX observations and our evolving heliosphere. Astrophys. J. Suppl. 203, 1.

McCracken, K.G., Ness, N.F.: 1966, The collimation of cosmic rays by the interplanetary magnetic field. J. Geophys. Res. 71, 3315-3318.

McCracken, K.G., McDonald, F.B., Beer, J., Raisbeck, G., Yiou, F.: 2004, A phenomenonological study of the long-term cosmic ray modulation, 850-1958 AD. J. Geophys. Res. 109, A12103.

Owens, M.J., Usoskin, I., Lockwood, M.: 2012, Heliospheric modulation of galactic cosmic rays during Grand Solar Maxima: Past and future variations. Geophys. Res. Lett. 39, 19102.

Owens, M.J., Lockwood, M., Barnard, L., Davis, C.J.: 2011, Solar Cycle 24: Implications for energetic particles and long-term space climate change. Geophys. Res. Lett. 38, L19106.

Paouris, E., Mavromichalaki, H., Belov, A., Guischina, R., Yanke, V.: 2012, Galactic cosmic ray modulation and the last solar minimum. Sol. Phys. 280, 255-271.

Parker, E.N.: 1965, The passage of energetically charged particles through interplanetary space. Planet. Space Sci. 13, 9-49.

Richardson, I.G.: 2004, Energetic particles and corotating interaction regions in the solar wind. Space Sci. Rev. 111, 267-376.

Richardson, I.G., Cane, H.V., Wibberenz, G.: 1999, A 22-year dependence in the size of nearecliptic corotating cosmic ray depressions during five solar minima. J. Geophys. Res. 104.

Rouillard, A., Lockwood, M.: 2007, The latitudinal effect of corotating interaction regions on galactic cosmic rays. Solar Phys. 245, 191-206.

Scott, C.J., Harrison, R.G., Owens, M.J., Lockwood, M., Barnard, L.: 2013, Solar wind modulation of UK lightning. Environ. Res. Lett. in press.

Smith, E.N.: 2001, The heliospheric current sheet. J. Geophys. Res. 106, 15819-15831.

Steinhilber, F., Beer, J., Frohlich, C.: 2009, Total solar irradiance during the past 9300 years inferred from the cosmogenic radionuclide Beryllium-10. AGU, Fall Meeting, GC24A-03.

Steinhilber, F., Abreu, J., Beer, J., McCracken, K.: 2010, Interplanetary magnetic field during the past 9300 years inferred from cosmogenic radionuclides. J. Geophys. Res. 115.

Thomas, B.T., Smith, E.J.: 1981, The structure and dynamics of the heliospheric current sheet. J. Geophys. Res. 86, 11105-11110.

Thomas, S.R., Owens, M.J., Lockwood, M.: 2013, The 22-year Hale Cycle in cosmic ray flux - Evidence for direct heliospheric modulation. Solar Phys. 289, 407-421.

Tritakis, V.P.: 1984, Heliospheric current sheet displacements during the Solar Cycle evolution. J. Geophys. Res 89, 6588-6598. 
Tsurutani, B.T., Gonzales, W.D., Gonzales, A.L.C., Tang, F., Arballo, J.K., Okada, M.: 1995, Interplanetary origin of geomagnetic activity in the declining phase of the Solar Cycle. $J$. Geophys. Res. 100, $21717-21734$.

Usoskin, I.G., Bazilevskaya, G.A., Kovaltsov, G.A.: 2011, Solar modulation parameter for cosmic rays since 1936 reconstructed from ground-based neutron monitors and ionization chambers. J. Geophys. Res. 116, A02104. 